\title{
Clinical Profile and Outcome of Patients with Acute Poisoning Admitted in Intensive Care Unit of Tertiary Care Center in Eastern Nepal
}

\author{
Lalit Kumar Rajbanshi, Batsalya Arjyal, Rakesh Mandal' \\ Depatment of Anesthesiology and Critcal Care Medicine, 'Department of Internal Medicine, Birat Medical College and Teaching Hospital, Biratnagar, Nepal
}

\section{Abstract}

Background: Acute poisoning is a common health-care problem requiring admission in the intensive care unit (ICU) in the developing countries. The study was conducted to assess the epidemiological parameters of acute poisoning and factors affecting clinical course and outcome of the patients in ICU in the eastern part of Nepal. Methodology: This was a prospective observational study conducted at ICU of tertiary care hospital in the eastern part of Nepal for the duration of 2 years. All the acute poisoning cases admitted in the ICU were studied for epidemiological profiles, poisoning characteristics, and various clinical factors affecting the outcome of the patients. Results: Eighty-five patients with acute poisoning were included in the study. Suicidal attempt leading to acute poisoning was seen in majority of the young population. Majority of the poisoning was due to the organophosphorous compounds (43.5\%). Patients with organophosphorous poisoning had a significantly longer duration of ICU stay $(P=0.020)$. The survival of the patients was significantly affected by age, duration of presentation to the hospital, Glasgow Coma Scale, Sequential Organ Failure Assessment score, need for mechanical ventilation, duration of ICU stay, hepatic failure, coagulopathy, and need for vasoactive drugs $(P<0.05)$. The independent predictors of mortality observed in the study were age, delayed presentation to the hospital, hepatic failure, and need for mechanical ventilation. Conclusion: Organophosphorous poisoning was the most common acute poisoning in young population leading to the ICU admission. Older age, delayed initiation of the resuscitative measures, need for mechanical ventilation, and hepatic failure significantly affected the mortality.

Keywords: Acute poisoning, intensive care unit, mortality, organophosphorous

\section{INTRODUCTION}

Acute poisoning is one of the major medical emergencies with significant morbidity and mortality. Most of the poisoning is due to the intention of deliberate self-harm. ${ }^{[1]}$ Accidental and occupational exposures to the pesticides leading to acute or chronic poisoning have been seen in farmers and children. The World Health Organization (WHO) have reported that around 0.3 million people die due to the various acute poisoning annually, out of which 200000 deaths are due to organophosphorous poisoning alone. ${ }^{[2,3]}$ The incidence is much more higher in the developing and resource-limited countries. Agricultural pesticides such as organophosphorous, organochloride, zinc, and aluminum phosphide are commonly used substances for intentional or accidental poisoning in Asian countries due to their easy availability while the misuse of

\begin{tabular}{|l|l|}
\hline \multicolumn{3}{c|}{ Access this article online } \\
\hline Quick Response Code: & Website: \\
\hline & www.ijccm.org \\
\hline
\end{tabular}

the drugs such as paracetamol, opioids, benzodiazepines, and tranquilizers is commonly seen in industrial and developed countries. $^{[4,5]}$

The epidemiological factors such as geography, occupation, socioeconomic status, literacy rate, and cultural and religious practices can influence the clinical presentation and outcome of the poisoning patients. This urges the need for periodical study for understanding the pattern of poisoning in a specific geographical area. Poisoning patients with the evidence of

Address for correspondence: Dr. Lalit Kumar Rajbanshi, Department of Anesthesiology and Critical Care Medicine, Birat Medical College and Teaching Hospital, Biratnagar, Nepal. E-mail: Ialitrajbanshi@gmail.com

This is an open access journal, and articles are distributed under the terms of the Creative Commons Attribution-NonCommercial-ShareAlike 4.0 License, which allows others to remix, tweak, and build upon the work non-commercially, as long as appropriate credit is given and the new creations are licensed under the identical terms.

For reprints contact: reprints@medknow.com

How to cite this article: Rajbanshi LK, Arjyal B, Mandal R. Clinical profile and outcome of patients with acute poisoning admitted in intensive care unit of tertiary care center in Eastern Nepal. Indian J Crit Care Med 2018;22:691-6. 
organ failure require admission to the intensive care unit (ICU) for organ support and specific management. There are various clinical entities that can determine the clinical course and outcome in the ICU. Besides the type of poison, delayed presentation and multi-organ failure that require immediately advanced life support organ can lead to high mortality. ${ }^{[6,7]}$

The eastern part of Nepal is a Terai belt where there is a wide variation in the socioeconomic status and cultural and religious practices. Various pesticides are easily available for agricultural purposes in the eastern of part Nepal. Few studies on epidemiology and clinical profile of poisoning have been conducted in Nepal, but most of them are confined to the central and western region and mainly observed on retrospective hospital-based data. ${ }^{[8,9]}$ However, little is known about the pattern of poisoning in the eastern part of Nepal. Furthermore, there is a paucity of studies on the clinical profile and mortality parameters for poisoning patients in ICU in Nepal.

Hence, this study was conducted with the objectives of evaluating epidemiological parameters for the acute poisons and factors affecting clinical course and outcome of the patients in ICU in the eastern part of Nepal.

\section{Methodology}

This was a prospective observational study conducted in the ICU of tertiary care hospital located at the eastern part of Nepal. The ethical approval for the study was obtained from the institutional ethical board, and an informed consent for the participation in the study was taken from the close relatives for each patient. The total duration of this study was 2 years starting from March 15, 2016, to March 15, 2018. During this study duration, all the cases of suspected acute poisoning admitted to the ICU were included in the study. A patient who got discharged, referred, left against medical advice or death within $24 \mathrm{~h}$ of ICU admission were excluded from the study. Immediately after admission, a detailed clinical history was taken and necessary therapeutic intervention was started without delay after a thorough clinical examination to stabilize the patients. Attempt for the prevention of the toxin absorption (gastric lavage and activated charcoal) was continued or started (if not started from the emergency department) in the patients presenting within $4 \mathrm{~h}$ of poisoning. Complete blood counts, urea, creatinine, liver function test including coagulation profile, serum electrolytes, random blood sugar, and toxicology screen for urine were routinely done for all the patients included in the study. Patients were managed with specific antidote if indicated.

Data regarding demographic profiles including age, sex, education-level socioeconomic status, and premorbid conditions were recorded from the patient or patient relatives. The information regarding the drug included the type of drug, route of ingestion, intention of poisoning, and duration of the first visit to the hospital. The type of poison was confirmed from the clinical presentation, circumstantial evidence, and the remaining container or packets brought by the patient relatives.
The clinical profile of the patients was assessed by recording Glasgow Coma Score (GCS) and Sequential Organ Failure Assessment (SOFA) score at the time of ICU admission, need for mechanical ventilation, need for vasoactive drugs, acute renal failure requiring renal replacement therapy, hepatic failure, and coagulopathy.

The patients were grouped into organophosphorous and nonorganophosphorous group. The nonorganophosphorous group included organochloride, zinc, and aluminum phosphide, drug toxicity, acute alcohol intoxication, and chemical poisoning. Although the organophosphorous and organochlorine compounds both are commonly used pesticides for agricultural purpose, the management of these two poisonings is different. The organophosphorous poisoning has specific antidote while organochlorine poisoning has symptomatic management only. However, the initial resuscitative measures which include taking care of airway, breathing and circulation, and attempt to decrease the absorption of the poison can be similar. The various clinical variables were compared between the two groups. The outcome of the patients was studied by assessing need of mechanical ventilation or any organ failure, duration of mechanical ventilation, duration of ICU stay, and mortality in the ICU.

All the patient data were recorded in Microsoft Excel and IBM SPSS statistics version 23 (Armonk, New York: IBM Corp) was used for data analysis. The study included continuous, discrete as well as categorical data. The continuous data were presented as mean and standard deviation while categorical data were presented as frequency and percentage. Chi-Square test and Mann-Whitney test were used for the analysis of categorical and continuous variables, respectively. Those variables, which were significant in the bivariate analysis, were subjected to the multivariate analysis. The stepwise backward logistic regression was used to find the adjusted effect of variables contributing mortality. $P<0.05$ was taken as statistically significant value.

\section{REsULTS}

A total of eighty-five patients with suspected or diagnosed acute poisoning due to various substances were included in the study. The consort diagram of the participants in the study is shown in Figure 1.

The demographic profile and patient characteristics were illustrated in Table 1. The incidence of poisoning was common in young adolescent age group (56.4\%) with female having a higher proportion of poisoning. Depression and chronic debilitating diseases such as end-stage renal failure, hepatic failure, malignancy, and HIV infection were observed to be associated with the poisoning.

The poisoning characteristics including mean time for exposure to the hospital, route of poisoning, and intention of poisoning are demonstrated in Table 2. In majority of the cases, the poisoning was ingested orally with the suicidal intention 


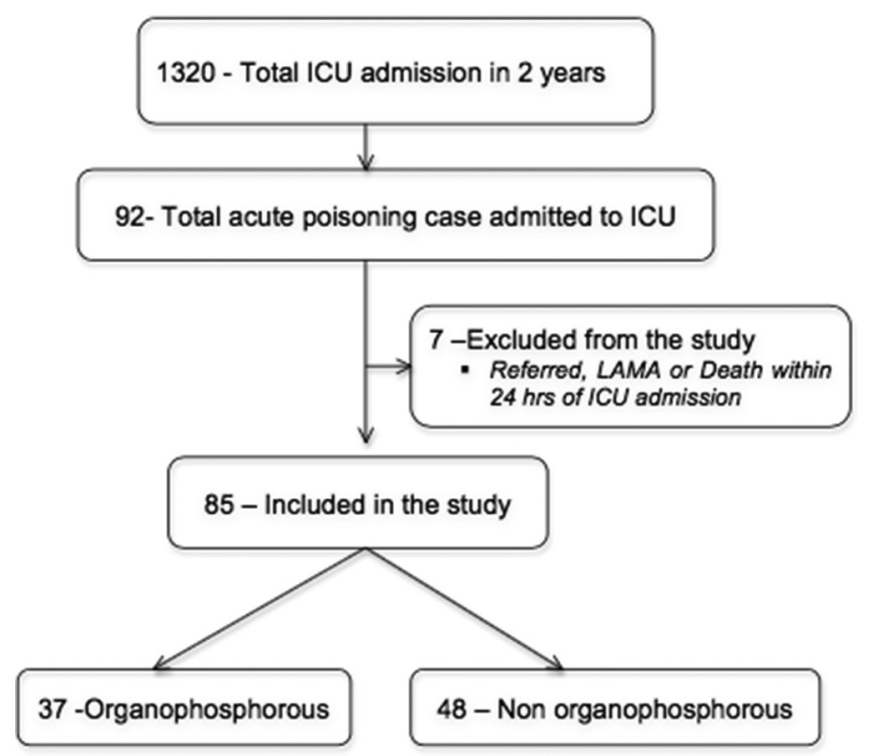

Figure 1: Consort diagram of the participants in the study

due to the suicidal attempt. Based on the availability of the toxicology screening kit, only 20 patients were subjected for urine toxicology screening where only 8 patients showed positive result for benzodiazepam and opioids.

The various types of compounds used for acute poisoning are shown in Figure 2. Majority of acute poisoning was due to the organophosphorous compounds (43.5\%). The various drugs used for poisoning were acetaminophen (paracetamol), salicylate (aspirin), benzodiazepams (diazepam), opioids (morphine), and amitriptyline. Similarly, the chemicals used for poisoning accidentally or intentionally were kerosene and acid used for the battery. In 5.8\% of patients, the type of poison could not be recognized.

As demonstrated in Table 3, the clinical and outcome variables were compared between the organophosphorous and nonorganophosphorous poisoning. The patients with nonorganophosphorous poisoning showed 1.6 times (odds ratio with 95\% confidence interval [CI]: 0.522-5.437) higher ICU mortality. Similarly, the patients with organophosphorous poisoning had a significantly longer duration of ICU stay $(P=0.020)$.

The observed mortality in the study population was $17.6 \%$ [Table 4]. The various clinical factors contributing mortality were studied and compared between survivors and nonsurvivors [Table 4]. Among the various factors studied, age, GCS, SOFA scoring, duration of ICU stay, duration for hospital presentation, need for mechanical ventilation, deranged coagulation profile, hepatic failure, and need for vasoactive drugs had a statistically significant impact on ICU mortality of the patients $(P<0.05)$. Out of all these significant variables, deranged coagulation profile and hepatic failure had a strong association for mortality $(P<0.001)$ with the significant odds ratio 3.80 (95\%CI: $0.59-11.45)$ and 4.24 (95\%CI: 0.74-10.61), respectively.

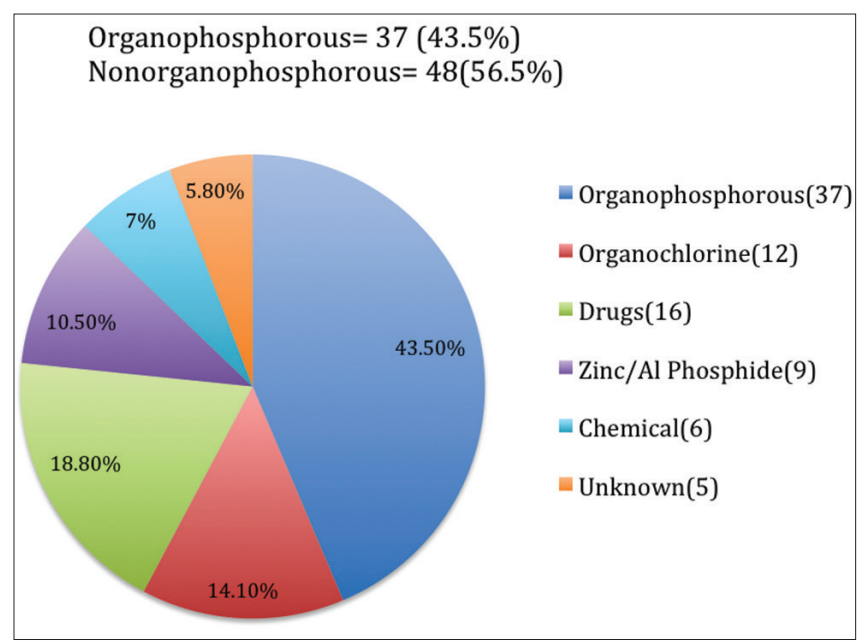

Figure 2: Types of poison

\begin{tabular}{lcc}
\hline \multicolumn{3}{l}{ Table 1: Demographic profile of poisoning patients } \\
\hline \multicolumn{1}{l}{ Variables } & $\boldsymbol{n}(\%)$ & Mean \pm SD \\
\hline Age (years) & $6(7)$ & $28.15 \pm 6.18(12-68)$ \\
$<15$ & $48(56.4)$ & \\
$15-30$ & $20(23.5)$ & \\
$30-50$ & $11(12.9)$ & Female: \\
$>50$ & & \\
Sex & $32(37.7)$ & \\
Male & $53(62.3)$ & \\
Female & & \\
Education & $9(10.5)$ & \\
Illiterate & $26(30.5)$ & \\
Primary & $32(37.6)$ & \\
Secondary & $18(21.1)$ & \\
Higher secondary & $7(8.2)$ & \\
Premorbid condition & $12(14.1)$ & \\
Psychiatric illness & \\
Chronic disease &
\end{tabular}

\section{Table 2: Poisoning characteristics}

\begin{tabular}{lc}
\hline Variables & Values \\
\hline Time for first exposure to hospital (mean $\pm \mathrm{SD}, \mathrm{h})$ & $3.62 \pm 1.41$ \\
Intention, $n(\%)$ & \\
Suicidal & $81(95.2)$ \\
Accidental & $3(3.5)$ \\
Homicidal & $1(1.1)$ \\
Route, $n(\%)$ & \\
Oral & $78(91.7)$ \\
Intravenous & $3(3.5)$ \\
Skin & $4(4.7)$ \\
Urine toxicology $(n=20)$ & Positive $=8$ \\
\hline SD: Standard deviation &
\end{tabular}

Multiple logistic regression, a backward logistic model, was used to assess the adjusted effect of all the statistically significant variables contributing ICU mortality [Table 5]. 
The model demonstrated that the most important factors contributing mortality were need of mechanical ventilation $(P=0.037)$, presence of hepatic failure $(P=0.019)$, duration of hospital presentation $(P=0.004)$, and age $(P=0.028)$.

\section{DISCUSSION}

Acute poisoning is a major health-care problem with a significant morbidity and mortality. It is a common medical emergency in the East Asian subcontinent where pesticides and insecticides are in easy access to the population. The prompt diagnosis and appropriate management is necessary for better outcome. However, the diagnosis and management can be challenged and complicated by the wide variation in the pattern of poisoning which depends on the various factors such as geographical area, socioeconomic status, literacy rate, age, and presence of various comorbid conditions. ${ }^{[6,10]}$

The mean age of presentation in the present study was comparable to the studies done by Zaheer et al., Gannur et al., and Nigam et al. where majority of the patients with acute poisoning presented within the age group of $16-30$ years. ${ }^{[11-13]}$

\begin{tabular}{|c|c|c|c|}
\hline Variables & OP (\%) & Non-OP (\%) & $P$ \\
\hline GCS & $9.5 \pm 3.6$ & $10.5 \pm 3.5$ & 0.220 \\
\hline SOFA & $4.3 \pm 2.5$ & $4.4 \pm 3.0$ & 0.778 \\
\hline Hospital presentation time & $3.2 \pm 1.9$ & $3.1 \pm 2.1$ & 0.725 \\
\hline Duration MV & $1.5 \pm 2.7$ & $1.2 \pm 2.0$ & 0.841 \\
\hline Duration ICU stay & $5.3 \pm 2.2$ & $4.2 \pm 1.9$ & 0.020 \\
\hline Mortality & $5(5.9)$ & $10(11.8)$ & 0.380 (OR 1.6) \\
\hline
\end{tabular}

OP: Organophosphorous; Non-OP: Nonorganophosphorous; GCS: Glasgow Coma Scale; SOFA: Sequential Organ Failure Assessment; MV: Mechanical ventilation; OR: Odds ratio; ICU: Intensive care unit
The young age presentation could be due to the fact that this age group was the most active age with a lot of personal and social responsibilities related with the personal career issues, studies, love affairs, and parental expectations. Female predominance for poisoning in our study can be explained by the fact that females are easily emotionally and mentally affected by the issues such as household violence and love affair failures. ${ }^{[13,14]}$

The duration of presentation to the hospital is an important factor to determine the clinical course and outcome. The patients presenting to the first health-care service within $2 \mathrm{~h}$ of acute poisoning have least morbidity and mortality. ${ }^{[15]}$ The delayed presentation of the patients in our study might be due to the various reasons such as delayed recognition of the victim after ingestion of the poison, difficult assess to the transport facility, or difficult access to the health-care centers. Delayed presentation after $4 \mathrm{~h}$ was also found by the work done by Ahuja et al. where they had mentioned that delayed initiation of resuscitative measures could be the possible contributing factor for the high mortality in their subjects. ${ }^{[7]}$

The finding that the oral route being the most common route of poisoning and suicidal intention being the most common mode of poisoning was also observed by Ahuja et al., Das, and Padmanabha et al.$^{[7,16,17]}$ Urine toxicology screening can be helpful for the identification of the toxins in limited cases. However, the negative result does not rule out the presence of toxins and as such does not have a significant impact on the management protocol. ${ }^{[6]}$ The test was done in 20 cases on the basis of clinical suspicion and availability of the kit at the time of presentation of the patient. Only eight patients had a positive result for diazepam and morphine.

As agriculture is the main occupation in the eastern part of Nepal, the organophosphorous compounds were easily available and were misused for self-intention harm. Many studies have demonstrated that the organophosphorous

\begin{tabular}{|c|c|c|c|c|c|c|}
\hline \multirow[t]{2}{*}{ Variables } & \multirow{2}{*}{$\begin{array}{c}\text { Survivor }(n=70 \text {, } \\
82.4 \%), n(\%)\end{array}$} & \multirow{2}{*}{$\begin{array}{c}\text { Nonsurvivor }(n=15 \text {, } \\
17.6 \%), n(\%)\end{array}$} & \multirow[t]{2}{*}{ OR } & \multicolumn{2}{|c|}{$95 \% \mathrm{Cl}$} & \multirow[t]{2}{*}{$P$} \\
\hline & & & & $\mathbf{L}$ & U & \\
\hline Age & $28.5 \pm 13.1$ & $38.4 \pm 15.1$ & & & & 0.012 \\
\hline GCS & $10.6 \pm 3.4$ & $7.7 \pm 3.5$ & & & & 0.005 \\
\hline SOFA & $3.9 \pm 2.4$ & $6.3 \pm 3.3$ & & & & 0.003 \\
\hline Duration of MV & $1.1 \pm 2.1$ & $2.4 \pm 3.0$ & & & & 0.069 \\
\hline Duration of ICU stay & $4.5 \pm 1.9$ & $5.8 \pm 2.7$ & & & & 0.041 \\
\hline Duration of presentation & $2.5 \pm 1.6$ & $6.0 \pm 1.5$ & & & & $<0.001$ \\
\hline Mechanical ventilation & $18(21.2)$ & $8(9.4)$ & 3.30 & 1.048 & 10.39 & 0.035 \\
\hline VAP & $6(7.1)$ & $3(3.5)$ & 2.66 & 0.58 & 12.15 & 0.192 \\
\hline ARF & $14(16.5)$ & $4(4.7)$ & 1.45 & 0.40 & 5.26 & 0.566 \\
\hline Coagulation & $4(4.7)$ & $6(7.1)$ & 3.80 & 0.59 & 11.45 & $<0.001$ \\
\hline Hepatic failure & $6(7.1)$ & $8(9.4)$ & 4.24 & 0.74 & 10.61 & $<0.001$ \\
\hline Vasoactive drugs & $18(21.2)$ & $10(11.8)$ & 5.77 & 1.74 & 19.18 & 0.002 \\
\hline $\mathrm{OP}$ & $32(37.65)$ & $5(5.9)$ & 1.68 & 0.522 & 5.437 & 0.380 \\
\hline Non-OP & $38(44.7)$ & $10(11.8)$ & & & & \\
\hline
\end{tabular}

GCS: Glasgow Coma Scale; SOFA: Sequential Organ Failure Assessment; VAP: Ventilator associated pneumonia; ARF: Acute renal failure; MV: Mechanical ventilation; OP: Organophosphorous; Non-OP: Nonorganophosphorous; OR: Odds ratio; ICU: Intensive care unit; L: Lower; U: Upper 
Table 5: Backward multiple logistic regression analysis showing clinical predictors of intensive care unit mortality

\begin{tabular}{lccc}
\hline Variables & $\boldsymbol{B}$ & SE & $\boldsymbol{P}$ \\
\hline Mechanical ventilation & 2.5 & 1.2 & 0.037 \\
Hepatic failure & 2.6 & 1.1 & 0.019 \\
Duration of presentation & 5.1 & 1.0 & 0.004 \\
Age & 2.57 & 1.17 & 0.028 \\
\hline SE Standard error & &
\end{tabular}

SE: Standard error

compound was the major toxin used in the cases presented with acute poisoning. ${ }^{[6,7,14]}$

The duration of ICU stay was significantly longer in patients with organophosphorous poisoning. The longer duration of mechanical ventilation in organophosphorous poisoning as observed in the study (though statistically nonsignificant) and the intermediate syndrome in this group of the patients might have contributed longer duration of ICU stay. Pulmonary complications such as increased secretion, pneumonia, and acute respiratory distress syndrome are frequently seen in patients with organophosphorous poisoning which leads to longer duration of stay in ICU. ${ }^{[18]}$

The mortality with acute poisoning in a well-established center with advanced life support is $1 \%-2 \%{ }^{[19]}$ The mortality has been seen higher in the center with limited critical care resources and with the delayed initiation of specific management. ${ }^{[19]}$ The mortality in our study was $17.6 \%$, which was comparable to the mortality observed in the study by Ahuja et al. (18\%) and Joshi and Patel (15.8\%). ${ }^{[7,20]}$ Aluminum phosphide and paracetamol poisoning along with the development of multi-organ failure might have contributed higher mortality in our study. Singh et al. had observed very low mortality in acute poisoning, i.e., $2.8 \%$, and this was due to the aluminum phosphide poisoning only ${ }^{[6]}$ The better outcome in this study was due to the well-equipped center with advanced life and organ support. ${ }^{[6]}$

Nonorganophosphorous poisoning patients had higher multi-organ failure and thus higher mortality (11.8\%). A study done by Mathai and Bhanu had demonstrated that factors responsible for poor prognosis were delayed presentation, early evidence of organ failure, acidosis, and need for vasoactive drugs for hemodynamically unstable patients. ${ }^{[21]}$ Ahuja et al had demonstrated higher mortality of the patients with high APACHEII score and high SOFA score. ${ }^{[7]}$ In the same study, the higher mortality was also observed in patients requiring mechanical ventilation and vasoactive support. ${ }^{[7]}$

Louriz et al. had found that need of vasoactive drugs for refractory shock was the independent factor for determining mortality. ${ }^{[22]}$ In the present study, all the statistically significant variables contributing poor prognosis were analyzed using multiple logistic regression to determine the independent predictors of mortality. Elderly age, need for mechanical ventilation, hepatic failure, and delayed presentation to the health-care center were the independent clinical predictors for contributing for higher mortality $(P<0.05)$. Initial resuscitative measures were delayed for the patients presenting late to the health-care center leading to early development of multi-organ failure and higher mortality.

\section{Limitation}

This is a single center-based study that makes data unreliable to represent the whole eastern part of Nepal. The epidemiological data do not include socioeconomic status, cultural and religious data, and occupational data that could have provided additional information regarding clinical spectrum of poisoning. Majority of the cases in the study were referred from other center where facility for advanced life support was not available. Minimizing the delay in initiating in advanced life support could have reduced the observed mortality in the study.

\section{ConcLusion}

Organophosphorous is the leading cause of acute poisoning requiring admission to the ICU in the eastern part of Nepal. Mortality is determined by the age of the patients, delayed presentation to the hospital, evidence of organ failure, need for mechanical ventilation, prolong duration of ICU stay, need for vasoactive drugs, and hepatic failure with coagulopathy. Hence, the study showed that initiation of early resuscitative measure and organ support can help to reduce the mortality of the poisoning patients.

\section{Financial support and sponsorship}

Nil.

\section{Conflicts of interest}

There are no conflicts of interest.

\section{References}

1. Konradsen F, Dawson AH, Eddleston M, Gunnell D. Pesticide self-poisoning: Thinking outside the box. Lancet 2007;369:169-70.

2. Thundiyil JG, Stober J, Besbelli N, Pronczuk J. Acute pesticide poisoning: A proposed classification tool. Bull World Health Organ 2008;86:205-9.

3. Eddleston M, Buckley NA, Eyer P, Dawson AH. Management of acute organophosphorus pesticide poisoning. Lancet 2008;371:597-607.

4. Marecek J. Culture, gender, and suicidal behavior in Sri Lanka. Suicide Life Threat Behav 1998;28:69-81.

5. McClure GM. Suicide in children and adolescents in England and wales 1970-1998. Br J Psychiatry 2001;178:469-74.

6. Singh O, Javeri Y, Juneja D, Gupta M, Singh G, Dang R, et al. Profile and outcome of patients with acute toxicity admitted in intensive care unit: Experiences from a major corporate hospital in urban India. Indian J Anaesth 2011;55:370-4.

7. Ahuja H, Mathai AS, Pannu A, Arora R. Acute poisonings admitted to a tertiary level Intensive Care Unit in Northern India: Patient profile and outcomes. J Clin Diagn Res 2015;9:UC01-4.

8. Mishra A, Shukla SK, Yadav MK, Gupta Ak. Epidemiological study of medicolegal organophosphorus poisoning in central region of Nepal. J Forensic Res 2012;3:1-5.

9. Dhakal AK, Shrestha D, Shakya A, Shah SC, Shakya H. Clinical profile of acute poisoning in children at a teaching hospital in Lalitpur. J Nepal Paediatr Soc 2014;34:100-3.

10. Abdollahi M, Jalali N, Sabzevari O, Hoseini R, Ghanea T. A retrospective study of poisoning in Tehran. J Toxicol Clin Toxicol 1997;35:387-93.

11. Zaheer MS, Aslam M, Gupta V, Sharma V, Khan SA. Profile of 
poisoning cases at a North Indian tertiary care hospital. Health Popul Perspect Issues 2009;32:176-83.

12. Gannur DG, Maka P, Narayan Reddy KS. Organophosphorus compound poisoning in Gulbarga region - A five year study. Indian J Forensic Med Toxicol 2008;2:3-11.

13. Nigam M, Jain AK, Dubey BP, Sharma VK. Trends of organophosphorus poisoning in Bhopal region an autopsy based study. JIAFM 2004;26:62-5.

14. Chintale KN, Patne SV, Chavan SS. Clinical profile of organophosphorus poisoning patients at rural tertiary health care centre. Int J Adv Med 2016;3:268-74.

15. Ramesha KN, Rao KB, Kumar GS. Pattern and outcome of acute poisoning cases in a tertiary care hospital in Karnataka, India. Indian J Crit Care Med 2009;13:152-5.

16. Das RK. Epidemiology of insecticide poisoning at A.I.I.M.S emergency service and role of its detection by gas liquid chromatographyin diagnosis. Medicoleg Update 2007;7:49-60.
17. Padmanabha TS, Gumma K, Kulkarni GP. Study of profile of organophpsphorus poisoning cases in a tertiary care hospital, North Karnataka, India. Int J Bio Sci 2014;5:332-9.

18. Giyanwani PR, Zubair U, Salam O, Zubair Z. Respiratory failure following organophosphate poisoning: A literature review. Cureus 2017;9:e1651.

19. Boukatta B, El Bouazzaoui A, Guemoune R, Houari N, Achour S, Sbai H. An epidemiological study of adult acute poisoning in Fez: Morocco. J Clin Toxicol 2014;4:1-5.

20. Joshi M, Patel DV. A study on clinical profile of patients with acute poisoning. GCSMC J Med Sci 2015;4:97-100.

21. Mathai A, Bhanu MS. Acute aluminium phosphide poisoning: Can we predict mortality? Indian J Anaesth 2010;54:302-7.

22. Louriz M, Dendane T, Abidi K, Madani N, Abouqal R, Zeggwagh AA, et al. Prognostic factors of acute aluminum phosphide poisoning. Indian J Med Sci 2009;63:227-34. 